\title{
Check-list de Malpighiaceae do estado de Mato Grosso do Sul
}

\author{
Augusto Francener ${ }^{1}$, Rafael Felipe de Almeida ${ }^{2} \&$ Renata Sebastiani $^{3}$
}

\author{
'Instituto de Botânica, Núcleo de Pesquisa Herbário do Estado, Av. Miguel Stéfano, 3687, CP 68041، \\ CEP 04045-972, Água Funda, São Paulo, SP, Brasil. augustofng@yahoo.com.br \\ ${ }^{2}$ Universidade Estadual de Feira de Santana, Departamento de Ciências Biológicas, Programa de Pós-Graduação em Botânica, \\ Avenida Transnordestina s/n, CEP44036-900, Novo Horizonte, Feira de Santana, BA, Brasil. \\ ${ }^{3}$ Universidade Federal de São Carlos, Centro de Ciências Agrárias, Campus de Araras, Via Anhanguera km 174, \\ CP 153, CEP 13699-970, Araras, SP, Brasil.
}

RESUMO - O objetivo do presente estudo foi apresentar o check-list das espécies de Malpighiaceae do estado do Mato Grosso do Sul. Para tanto, foram realizadas viagens de campo e consultas às coleções ou os bancos de dados referentes a 30 herbários. Registramos 118 espécies de Malpighiaceae, representando um acréscimo de 30\% a listagem anterior para este estado (86 espécies). Os gêneros mais numerosos em espécies foram Heteropterys Kunth (21), Byrsonima Rich. ex. Kunth (15) e Banisteriopsis C.B. Rob. (15), enquanto oito gêneros foram representados por apenas uma espécie cada. O bioma Cerrado apresenta a maior diversidade de Malpighiaceae (95 espécies), seguido pelo Pantanal (37 espécies) e Floresta Atlântica (14 espécies). Por outro lado, 30 espécies são novas ocorrências para este estado e nove espécies foram consideradas ameaçadas de extinção. Esses dados evidenciam a importância do estado do Mato Grosso do Sul para a conservação da diversidade de Malpighiaceae no Brasil.

Palavras-chave: Banisteriopsis, Byrsonima, Cerrado, Flora neotropical, Heteropterys, Malpighiales

ABSTRACT - Checklist de Malpighiaceae do estado de Mato Grosso do Sul. The goal of this study was to present a checklist of Malpighiaceae species from the state of Mato Grosso do Sul. We performed field trips and visits to ca. 30 Brazilian herbaria. A total of 118 species of Malpighiaceae were recorded for the state of Mato Grosso do Sul, representing 30\% new records for this state (86 species previously reported). The richest genera were Heteropterys Kunth (21), Byrsonima Rich. ex. Kunth (15) and Banisteriopsis C.R. Rob. (15), while eight genera were represented by a single species each. The Cerrado biome shows the greatest diversity of Malpighiaceae (95 species) when compared to the Pantanal wetland ( 37 species) and the Atlantic Forest (14 species). On the other hand, 30 species are new records for this state, and nine species were considered endangered. These results evidence the importance of Mato Grosso do Sul state in to preserve the biodiversity of Malpighiaceae in Brazil.

Keywords: Banisteriopsis, Byrsonima, Cerrado, Heteropterys, Malpighiales, Neotropical Flora

\section{INTRODUÇÃO}

Malpighiaceae possui cerca de 77 gêneros e 1300 espécies de distribuição pantropical, sendo $85 \%$ de sua diversidade encontrada principalmente em áreas tropicais e subtropicais da região Neotropical (Davis et al. 2002, Davis \& Anderson 2010). No Brasil, ocorrem 44 gêneros e ca. 561 espécies distribuídas por todos os biomas do país (BFG 2015), sendo o Cerrado o mais diverso em espécies e seu provável centro de diversidade (Anderson 1979, Sebastiani et al. 2015).

A família é caracterizada pela presença de folhas simples opostas, em geral com nectários extraflorais de distribuição bastante variável e tricomas unicelulares em formato de T, V ou Y. Suas sépalas laterais frequentemente apresentam duas glândulas secretoras de óleo na face abaxial, as quais são frequentemente ausentes na sépala anterior, e suas pétalas são geralmente unguiculadas, com a pétala posterior geralmente ereta e diferenciada das demais. $\mathrm{Na}$ maioria das espécies o androceu é formado por dois verticilos de estames, iguais ou desiguais, e o gineceu apresenta três carpelos, livres ou fusionados, cada um contendo um estilete. Seus frutos são drupas, nozes ou mais frequentemente esquizocárpicos, os quais dividem-se em três mericarpos lisos, alados ou setosos (Almeida et al. 2016, Anderson 1981).

O estado do Mato Grosso do Sul (MS) apresenta cerca de 68 espécies de Malpighiaceae (Anderson 1998) ocorrendo em seus três diferentes biomas: Pantanal, Cerrado e Floresta Atlântica, os dois últimos considerados hotspots mundiais prioritários à conservação (MMA 2007, Mittermeir et al. 2005), além de fragmentos de Chaco. De acordo com dados recentes, são estimados cerca de 21 gêneros e 70 espécies de Malpighiaceae para o MS, sendo que 63 destas são registradas para o Cerrado e apenas seis para o Pantanal (BFG 2015). Além das discrepâncias quanto a sua diversidade de espécies de Malpighiaceae, o MS apresenta os menores índices de esforço amostral de coletas no Centro-Oeste brasileiro registrado em literatura (Gomes-Klein \& Rizzo 2006).

Assim, um maior esforço de coletas aliado a uma análise das coleções de Malpighiaceae do MS em herbários nacionais e internacionais pode revelar um novo panorama da diversidade da família no estado. Dessa forma, o 
presente estudo teve como objetivo apresentar um checklist atualizado de Malpighiaceae para o estado do Mato Grosso do Sul, além de uma análise do esforço amostral desta família nos municípios e biomas deste estado.

\section{Principais grupos de pesquisa}

Atualmente há quatro especialistas em Malpighiaceae diretamente envolvidos em estudos taxonômicos no Brasil, Dr. André Amorim (Professor da Universidade Estadual de Santa Cruz e Curador do Herbário CEPEC, Bahia), Dra. Maria Candida Henrique Mamede (Pesquisador Científico VI e Curadora do Herbário SP, Instituto de Botânica, São Paulo), Dra. Renata Sebastiani (Professora da Universidade Federal de São Carlos, São Paulo) e Dr. Augusto Francener (Universidade Federal do Rio Grande do Norte). Cada especialista desenvolve pesquisas individuais em Malpighiaceae em suas respectivas instituições, bem como estudos em conjunto, principalmente para a elaboração da Flora do Brasil 2020 (Almeida pers. obs., Almeida et al. 2013, Almeida et al. 2016, Mamede et al. 2010, Wanderley et al. 2012, Sebastiani et al. 2015). Nesse sentido, é esperado que esses especialistas continuem participando ativamente de levantamentos de Malpighiaceae em várias regiões do país, em orientações de trabalhos de Iniciação Científica e de Pós-Graduação e em estudos taxonômicos e filogenéticos em grupos problemáticas, como os gêneros Byrsonima, Heteropterys, Mascagnia e segregados, além da tribo Gaudichaudieae.

\section{Principais Lacunas de Conhecimento}

Vários gêneros de Malpighiaceae vêm sendo recentemente estudados, como Alicia W.R. Anderson (Anderson 2006), Amorimia W.R. Anderson (Almeida et al. in prep.), Aspicarpa Rich. (Anderson 2014), Banisteriopsis C.R. Rob. ex Small (Gates 1982, Carvalho et al. 2010, Almeida \& Mamede submetido), Barnebya (Anderson \& Gates 1981), Bronwenia W.R. Anderson \& C. Davis (Carvalho et al. 2010, Almeida \& Mamede submetido), Bunchosia (Anderson \& Anderson 2015, Almeida \& Pellegrini 2016), Byrsonima (Mamede 1981, Anderson 1982, Anderson 1993, Elias 2005, Francener 2016), Camarea A. St.-Hil. (Mamede 1990), Coleostachys A. Juss. (Almeida \& Hall in prep.), Diplopterys A. Juss. (Anderson \& Davis 2006, Carvalho et al. 2010, Almeida \& Mamede submetido), Galphimia Cav. (C. Anderson 2007), Heteropterys (Amorim 2002, Amorim 2003, Amorim 2009, Anderson 1982, Anderson 1993, Anderson 1997, Anderson 1999, Anderson 2014, Sebastiani \& Mamede 2010, Pessoa \& Amorim 2015, 2016), Hiraea (C. Anderson 2013a, C. Anderson 2013b, C. Anderson 2014), Janusia A. Juss. (Sebastiani 2010, Sebastiani \& Mamede 2014, Almeida \& Mamede submetido), Lophanthera (Anderson 2014), Mcvaughia (Amorim \& Almeida 2015), Mascagnia Bertero ex. Colla (Anderson \& Davis 2005, Anderson \& Davis 2012, 2013, Anderson 2014, C. Anderson 2001), Peixotoa A. Juss. (C. Anderson 1982, Almeida \& Mamede submetido), Pterandra (C. Anderson 1997b, C. Anderson
2005) Stigmaphyllon A. Juss. (Almeida 2015, Almeida \& Amorim 2014, 2015, Almeida \& Mamede submetido, Almeida et al. 2015, C. Anderson 1997a, C. Anderson 2009, C. Anderson 2011, Lima et al. 2015), Tetrapterys Cav. (Francener et al. 2015, Almeida et al. in prep.) e Thryallis Mart. (C. Anderson 1995). Outros gêneros, ainda, têm sido recentemente estabelecidos, em geral com base em análises filogenéticas a partir de dados moleculares e morfológicos, como em Aenigmatanthera W.R. Anderson, Alicia W.R. Anderson, Amorimia W.R. Anderson, Callaeum Small., Christianella W.R. Anderson, Niedenzuella W.R. Anderson (Anderson 2006) e Bronwenia (Anderson \& Davis 2007). No entanto, gêneros como Aspicarpa Rich., Hiraea Jacq. e Mascagnia ainda necessitam de revisões taxonômicas e estudos filogenéticos mais detalhados para melhor delimitação de suas espécies.

Algumas floras de Malpighiaceae foram realizadas no país até o presente, dentre as quais podemos citar: Flora do Pico das Almas (Anderson 1995), Flora da Reserva Biológica de Macaé de Cima (Amorim 1994), Flora da Serra do Cipó (Mamede 1987), Flora de Grão-Mogol (Mamede 2004), Malpighiaceae do estado da Bahia (Almeida et al. in prep.), Malpighiaceae do estado do Espírito Santo (Almeida \& Mamede 2014), Malpighiaceae do estado do Paraná (Almeida \& Amorim 2014), Malpighiaceae do estado do Rio de Janeiro (Silva \& Almeida 2015), Malpighiaceae do estado do Sergipe (Almeida et al. 2015), Malpighiaceae da Chapada dos Veadeiros (Sebastiani et al. 2015), Malpighiaceae da restinga da Marambaia, Rio de Janeiro (Almeida et al. in prep.), Malpighiaceae da Serra Geral de Licínio de Almeida (Almeida et al. in prep.), Malpighiaceae do cerrado do Parque Estadual do Mirador, Maranhão (Conceição et al. 2011), Malpighiaceae da Reserva Ducke (Vicentini 1999), Malpighiaceae das restingas do Pará (Alexandrino et al. 2011), Malpighiaceae da Ilha do Cardoso (Mamede 1992) e Malpighiaceae do Parque Estadual das Fontes do Ipiranga (Mamede 1984).

\section{Principais Acervos}

Os maiores herbários do país abrigam as principais coleções de Malpighiaceae, como os herbários R e RB. Grande número de tipos da família estão depositados nestes herbários, com o herbário $\mathrm{R}$ ainda possuindo ainda uma importante coleção histórica, que já foi consultada por vários especialistas do país e do exterior, como o Dr. Franz Niedenzu e Dr. William R. Anderson. Ainda, os herbários CEPEC, HUEFS e SP possuem coleções expressivas de Malpighiaceae devido à especialistas associados, bem como os herbários MBM e UB que mantinham um întercâmbio contínuo espécimes com o herbário $\mathrm{MICH}$, devido aos estudos do Dr. William Anderson.

\section{Perspectiva para o Grupo nos próximos 10 anos}

As perspectivas de trabalho dos principais grupos de pesquisa em Malpighiaceae no país são: reconstrução de filogenias moleculares para a tribo Gaudichaudieae, e para os gêneros Amorimia (Almeida et al. in prep.), Banisteriopsis 
(Almeida et al. in prep.), Byrsonima (Francener et al. in prep.), Stigmaphyllon (Almeida et al. in prep.) e Tetrapterys s.l. (Almeida et al. in prep.); conclusão das monografias de Malpighiaceae para os estados da Bahia, Espírito Santo, Goiás, Tocantins, São Paulo e Sergipe; além da elaboração da monografia de Malpighiaceae para a Flora do Brasil 2020 e guias de campo.

\section{MATERIAL E MÉTODOS}

Para a elaboração da presente lista foram analisadas as coleções obtidas durante viagens de campo bem como aquelas depositadas nos herbários ALCB, ASE, BHCB, CEPEC, CGMS, CPAP, COR, ESA, FUEL, HSJRP, HUEFS, HUESC, HUFU, HUM, INPA, IPA, MBM, MBML, RB, SP, SPF, SPSF, UB, UEC, UFMS, UPCB, US, VIES (acrônimos segundo Thiers 2016). Foram também analisadas as informações de coleções determinadas por especialistas em Malpighiaceae contidas em bancos de dados da rede brasileira de herbários, obtidas pelo Sistema de Informações SpeciesLink (2016). Para cada espécie foi selecionada uma coleção, dando-se preferência às coleções pessoalmente analisadas e recentemente coletadas. Os mapas foram elaborados no programa $\operatorname{ArcGis} \AA$ a partir de shape files obtidos no site do Ministério do Meio Ambiente (2012) e de informações contidas em todas as coleções de Malpighiaceae disponíveis para o Mato Grosso do Sul, mesmo aquelas não identificadas em nível de espécie. Para a delimitação dos biomas ocorrentes no MS seguiu-se a classificação proposta pelo Ministério do Meio Ambiente (2007) que inclui os fragmentos de Chaco como parte do Bioma Pantanal.

\section{RESULTADOS E DISCUSSÃO}

Foram contabilizadas cerca de 1360 coleções, representando 118 espécies e 26 gêneros de Malpighiaceae para o Mato Grosso do Sul (Tab. 1). Deste total, 30 são novas ocorrências, representando um acréscimo de $25 \%$ ao número de espécies anteriormente citado para a área de estudo (Anderson 1998, BFG 2015). Esses dados já foram atualizados na última versão da Flora do Brasil Online, visando sua incorporação na Flora do Brasil Online 2020 (Mamede et al. 2015; BFG 2015). Os principais acervos contendo coleções recentemente determinadas de Malpighiaceae para o estado estão nos herbários CGMS, MBM e SP. Os gêneros mais diversos foram Heteropterys (21 espécies), Byrsonima (15 espécies) e Banisteriopsis (15 espécies), enquanto gêneros como Aenigmatanthera, Alicia, Amorimia, Callaeum, Carolus, Christianella, Dicella Griseb. e Heladena A. Juss. foram representados apenas por uma espécie cada.

O bioma com maior diversidade foi o Cerrado, com 95 espécies, seguido do Pantanal com 37 espécies e da Floresta Atlântica com 14 espécies (Fig.1). O Cerrado brasileiro possui 223 espécies de Malpighiaceae, sendo o Cerrado sul-mato-grossense abrange $44 \%$ deste total.
Para o Pantanal brasileiro era relatada a ocorrência de somente seis espécies de Malpighiaceae (BFG 2015), sendo o Pantanal sul-mato-grossense responsável pelo acréscimo de 32 novos registros para este bioma no país. A Floresta Atlântica sul-mato-grossense, por sua vez, apresentou uma baixa diversidade de espécies da família (14 espécies) quando comparada com aquela de outros estados inseridos neste bioma (Wanderley et al. 2012, Almeida et al. 2016), provavelmente devido ao baixo esforço amostral nas áreas ocupadas por esse bioma no Mato Grosso do Sul (Fig. 3).

Os municípios com maior esforço amostral para Malpighiaceae foram Corumbá (374 espécimes), Campo Grande (310) e Três Lagoas (180) (Fig. 3). Municípios como Alcinópolis, Angélica, Aparecida do Taboado, Ivinhema, Nova Alvorada do Sul, dentre outros, não possuem coleções de Malpighiaceae nos herbários visitados e consultados, além de estarem inseridos no domínio do Cerrado e da Floresta Atlântica, hotspots mundiais prioritários à conservação (Mittermeier et al. 2005) (Fig. 2).

Aspicarpa salicifolia (Chodat.) Nied., Bunchosia argentea (Jacq.) DC., Camarea ericoides A. St.-Hil., Dicella bracteosa (A. Juss.) Griseb., Heteropterys bicolor A. Juss., H. macradena (DC.) W.R. Anderson, H. rubiginosa A. Juss., H. syringifolia Griseb., H. umbellata A.Juss., Tetrapterys chamaecerasifolia A. Juss., T. microphylla (A. Juss.) Nied. e T. salicifolia (A. Juss.) Nied. são referidas por Anderson (1998) e ou Mamede et al. (2010 e 2015) para o estado do Mato Grosso do Sul. No entanto, estas espécies não foram incluídas neste trabalho, uma vez que não foram localizadas coleções das mesmas para o Mato Grosso do Sul nos herbários consultados e em herbários virtuais. As espécies Bunchosia armeniaca (Cav.) DC., B. glandulifera (Jacq.) H.B.K., Lophanthera lactescens Ducke, Malpighia glabra L. e M. emarginata DC. Também não são citadas pois, apesar de serem registradas para o estado, são espécies amplamente cultivadas. Galphimia brasiliensis (L.) A. Juss. também foi considerada como cultivada, apesar de sua ocorrência natural ser relatada para o Mato Grosso do Sul por Anderson (1998). No entanto, em revisão recente deste gênero, C. Anderson (2007) incluiu a coleção de G. brasiliensis citada por Anderson (1998) para o Mato Grosso do Sul em G. australis e limitou a distribuição de G. brasiliensis à região Nordeste do Brasil.

O estado do Mato Grosso do Sul ainda não dispõe de uma lista de espécies vegetais ameaçadas. Porém, dentre as espécies referidas para a área de estudo, nove aparecem no Livro Vermelho das Espécies Vegetais Ameaçadas do estado de São Paulo (Mamede et al. 2007). Quatro são consideradas quase ameaçadas, por terem ocorrência desconhecida em unidades de conservação, baixa densidade populacional e ocorrerem exclusivamente em um tipo de formação vegetal, $A$. pulchella, B. basiloba, $H$. campestres e $J$. mediterranea. No entanto, de acordo com Sebastiani (2010), J. mediterranea ocorre em várias formações vegetais, tais como formações de Cerrado, Floresta Semidecidual e até áreas degradadas. Três espécies são consideradas vulneráveis por terem distribuição restrita no 
Tabela 1. Lista das espécies de Malpighiaceae para o estado do Mato Grosso do Sul. Novas ocorrências $\left({ }^{*}\right)$; $\left({ }^{1}\right)$ citações para o estado com base em Anderson (1998); $\left({ }^{2}\right)$ citações para o estado baseadas em BFG (2015). Biomas: $\mathrm{C}=\mathrm{Cerrado}$; $\mathrm{F}=\mathrm{Floresta}$ Atlântica; $\mathrm{P}=\mathrm{Pantanal}$; NE = número de espécimes localizados para o presente estudo

\begin{tabular}{|c|c|c|c|}
\hline Espécie & Voucher & Biomas & $\mathrm{NE}$ \\
\hline Aenigmatanthera lasiandra (A. Juss.) W.R. Anderson * & Francener $1182(\mathrm{SP})$ & $\mathrm{C}$ & 2 \\
\hline Alicia anisopetala (A. Juss.) W.R. Anderson ${ }^{1}$ & Hatschbach 58655 (MBM) & $\mathrm{F}$ & 8 \\
\hline Amorimia pubiflora (A. Juss.) W.R. Anderson ${ }^{2}$ & Sebastiani 324 (SP) & $\mathrm{C}, \mathrm{F}, \mathrm{P}$ & 6 \\
\hline Aspicarpa sericea Griseb. ${ }^{1}$ & Magalhães 17 (CGMS) & $\mathrm{C}, \mathrm{P}$ & 3 \\
\hline A. pulchella (Griseb.) O’Donell \& Lourteig ${ }^{2}$ & Damasceno Jr. 1306 (SP) & $\mathrm{C}, \mathrm{P}$ & 4 \\
\hline Banisteriopsis acerosa (Nied.) B. Gates ${ }^{1,2}$ & Hatschbach 62171 (MBM) & $\mathrm{C}$ & 9 \\
\hline B. argyrophylla (A. Juss.) B. Gates ${ }^{1,2}$ & Caliente $140(\mathrm{SP})$ & $\mathrm{C}$ & 18 \\
\hline B. campestris (A. Juss.) Little ${ }^{1,2}$ & Anunciação 729 (SP) & $\mathrm{C}$ & 14 \\
\hline B. confusa B. Gates ${ }^{1,2}$ & Barbosa 1968 (SP) & $\mathrm{C}$ & 11 \\
\hline B. gardneriana (A. Juss.) W.R. Anderson \& B. Gates ${ }^{2}$ & Caliente 521 (SP) & $\mathrm{C}$ & 3 \\
\hline B. laevifolia (A. Juss.) B. Gates ${ }^{1,2}$ & Pott 12817 (SP) & $\mathrm{C}$ & 36 \\
\hline B. malifolia (Nees \& Mart.) B. Gates* & Hatschbach 76254 (MBM) & $\mathrm{C}$ & 9 \\
\hline B. megaphylla (A. Juss.) B. Gates ${ }^{1}$ & Hatschbach 33981 (UB) & $\mathrm{C}$ & 9 \\
\hline B. muricata (Cav.) Cuatrec. ${ }^{1,2}$ & Pott $12790(\mathrm{SP})$ & $\mathrm{C}$ & 11 \\
\hline B. nummifera (A. Juss.) B. Gates ${ }^{2}$ & Pott 11297 (SP) & $\mathrm{C}$ & 3 \\
\hline B. oxyclada (A. Juss.) B. Gates ${ }^{2}$ & Jacques 754 (SP) & $\mathrm{C}$ & 17 \\
\hline B. paraguariensis $\mathrm{B}$. Gates ${ }^{1}$ & Hatschbach 45881 (MBM) & $\mathrm{C}$ & 7 \\
\hline B. pulchra B. Gates var. pulchra ${ }^{1}$ & Hatschbach 73030 (MBM) & $\mathrm{C}$ & 4 \\
\hline B. stellaris (Griseb.) B. Gates ${ }^{1,2}$ & Pott 2206 (SP) & $\mathrm{C}, \mathrm{P}$ & 22 \\
\hline B. variabilis $\mathrm{B}$. Gates ${ }^{1,2}$ & Pott 7754 (SP) & $\mathrm{C}$ & 6 \\
\hline Bronwenia longipilifera (B. Gates) W.R. Anderson \& C. Davis ${ }^{1,2}$ & Ribas 2538 (MBM) & $\mathrm{P}$ & 2 \\
\hline B. megaptera (B. Gates) W.R. Anderson \& C. Davis* & Hatschbach 65674 (MBM) & $\mathrm{C}$ & 1 \\
\hline Bunchosia pallescens Skottsb. ${ }^{1}$ & Hatschbach 51611 (MBM) & $\mathrm{C}$ & 7 \\
\hline B. paraguariensis Nied. ${ }^{1}$ & Francener 878 (CGMS) & $\mathrm{C}, \mathrm{P}$ & 20 \\
\hline Byrsonima affinis W.R. Anderson ${ }^{1,2}$ & Santos $5258(\mathrm{SP})$ & $\mathrm{C}$ & 22 \\
\hline B. arthropoda A. Juss. * & Tozzi 143 (SP) & $\mathrm{C}$ & 3 \\
\hline B. basiloba A. Juss. ${ }^{2}$ & Tavares $1484(\mathrm{SP})$ & $\mathrm{C}$ & 38 \\
\hline B. chrysophylla Kunth ${ }^{2}$ & Oliveira 163 (CGMS) & $\mathrm{C}$ & 2 \\
\hline B. clausseniana A. Juss. * & Damasceno Jr. 2594 (CGMS) & $\mathrm{C}$ & 2 \\
\hline B. coccolobifolia Kunth $^{1,2}$ & Cordeiro 1037 (SP) & $\mathrm{C}$ & 60 \\
\hline B. cydoniifolia A. Juss. ${ }^{1,2}$ & Francener 805 (CGMS) & $\mathrm{C}, \mathrm{P}$ & 66 \\
\hline B. intermedia A. Juss. ${ }^{1,2}$ & Francener 803 (CGMS) & $\mathrm{C}, \mathrm{P}, \mathrm{F}$ & 180 \\
\hline B. laxiflora Griseb. ${ }^{2}$ & Pott $4510(\mathrm{SP})$ & $\mathrm{C}, \mathrm{P}$ & 10 \\
\hline B. pachyphylla A. Juss. ${ }^{1,2}$ & Francener 871 (CGMS) & $\mathrm{C}$ & 41 \\
\hline 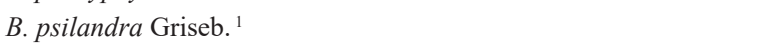 & Hatschbach 51534 (MBM) & $\mathrm{C}$ & 7 \\
\hline B. rigida A. Juss. ${ }^{1}$ & Damasceno Jr. 4438 (CGMS) & $\mathrm{C}$ & 5 \\
\hline B. sericea DC. ${ }^{1}$ & Anderson 11265 (SP) & $\mathrm{C}$ & 1 \\
\hline B. umbellata A. Juss. ${ }^{1,2}$ & Pott 5675 (SP) & $\mathrm{C}, \mathrm{P}$ & 17 \\
\hline B. verbascifolia (L.) DC. ${ }^{1,2}$ & Nascimento 1417 (SP) & $\mathrm{C}$ & 42 \\
\hline Callaeum psilophyllum (A. Juss.) D.M. Johnson ${ }^{1,2}$ & Pott $6159(\mathrm{SP})$ & $\mathrm{C}, \mathrm{P}$ & 11 \\
\hline Camarea affinis A. St.-Hil. ${ }^{1,2}$ & Barros $980(\mathrm{SP})$ & $\mathrm{C}$ & 4 \\
\hline C. hirsuta A. St.-Hil.* & Damasceno Jr. 4591 (CGMS) & $\mathrm{C}$ & 1 \\
\hline Carolus chlorocarpus (A. Juss.) W.R. Anderson ${ }^{2}$ & Pott 4908 (SP) & $\mathrm{C}, \mathrm{P}$ & 4 \\
\hline Christianella multiglandulosa (Nied.) W. R. Anderson* & Francener 873 (CGMS) & $\mathrm{C}$ & 51 \\
\hline Dicella macroptera A. Juss. ${ }^{1}$ & Jacques 743 (SP) & $\mathrm{C}, \mathrm{P}$ & 18 \\
\hline Diplopterys amplectescens (B. Gates) W.R. Anderson \& C. Davis* & Pott 9236 (CGMS) & $\mathrm{C}$ & 1 \\
\hline D. lutea (Griseb.) W.R. Anderson \& C. Davis ${ }^{1}$ & Matos-Alves 191 (CGMS) & $\mathrm{C}, \mathrm{F}, \mathrm{P}$ & 9 \\
\hline D. pubipetala (A. Juss.) W.R. Anderson \& C. Davis ${ }^{1,2}$ & Francener 868 (CGMS) & $\mathrm{C}$ & 59 \\
\hline D. valvata (W.R. Anderson \& B. Gates) W.R. Anderson \& C. Davis* & Hatschbach 74743 (MBM) & $\mathrm{C}$ & 3 \\
\hline Galphimia australis Chodat $^{1,2}$ & Francener 891 (CGMS) & $\mathrm{C}, \mathrm{P}$ & 19 \\
\hline G. platyphylla Chodat $^{2}$ & Hatschbach 33058 (NY) & $\mathrm{C}$ & 1 \\
\hline Heladena multiflora (Hook. \& Arn.) Nied. ${ }^{1}$ & Hatschbach 48489 (MBM) & $\mathrm{C}$ & 1 \\
\hline Heteropterys amplexicaulis Morong ${ }^{1}$ & Klein 2158 (RB) & $\mathrm{C}$ & 1 \\
\hline H. argyrophaea A. Juss. ${ }^{2}$ & Hatschbach 74251 (SP) & $\mathrm{C}$ & 15 \\
\hline H. byrsonimiifolia A. Juss. ${ }^{1,2}$ & Ribas 4 (CGMS) & $\mathrm{C}$ & 8 \\
\hline H. campestris A. Juss. ${ }^{2}$ & Pott 200 (SP) & $\mathrm{C}$ & 6 \\
\hline
\end{tabular}


Tabela 1. Cont.

\begin{tabular}{|c|c|c|c|}
\hline Espécie & Voucher & Biomas & $\mathrm{NE}$ \\
\hline H. cochleosperma A. Juss. ${ }^{1,2}$ & Sebastiani 322 (SP) & $\mathrm{C}, \mathrm{F}, \mathrm{P}$ & 16 \\
\hline H. coriacea A. Juss. * & Barros 1167 (SP) & $\mathrm{C}$ & 14 \\
\hline H. corumbensis Kuntze $^{1}$ & Chagas Silva 792 (FUEL) & $\mathrm{P}$ & 1 \\
\hline H. dumetorum (Griseb.) Nied. * & Tiritan 92 (SP) & $\mathrm{C}, \mathrm{P}$ & 6 \\
\hline H. eglandulosa A. Juss. ${ }^{2}$ & Penha 22 (CGMS) & $\mathrm{C}$ & 5 \\
\hline H. escalloniifolia A. Juss. * & Hatschbach 51589 (MBM) & $\mathrm{C}$ & 2 \\
\hline H. glabra Hook. \& Arn. ${ }^{1}$ & Pott 4415 (SP) & $\mathrm{C}, \mathrm{P}$ & 13 \\
\hline H. grandiflora A. Juss. * & Hatschbach 32520 (SP) & $\mathrm{C}$ & 2 \\
\hline H. hatschbachii W.R. Anderson ${ }^{1,2}$ & Anderson 11257 (MBM) & $\mathrm{C}$ & 2 \\
\hline H. hypericifolia A. Juss. * & Francener 942 (CGMS) & $\mathrm{C}, \mathrm{P}$ & 18 \\
\hline H. nervosa A. Juss. ${ }^{1}$ & Catharino 1905 (SP) & $\mathrm{C}$ & 10 \\
\hline H. orinocensis (Kunth) A. Juss. * & Moreira 371 (CGMS) & $\mathrm{C}$ & 1 \\
\hline H. procoriacea Nied. $^{2}$ & Hatschbach 32513 (SP) & $\mathrm{C}$ & 7 \\
\hline H. pteropetala A. Juss. ${ }^{1}$ & Barbosa-Filho 86 (CGMS) & $\mathrm{C}$ & 7 \\
\hline H. rhopalifolia A. Juss. ${ }^{1}$ & Francener 880 (CGMS) & $\mathrm{C}$ & 19 \\
\hline H. sylvatica A. Juss. ${ }^{1,2}$ & Damasceno Jr. 2869 (SP) & $\mathrm{C}$ & 3 \\
\hline H. tomentosa A. Juss. ${ }^{1}$ & Cunha 340 (CGMS) & $\mathrm{C}, \mathrm{P}$ & 26 \\
\hline Hiraea cuiabensis Griseb. * & Pott 8206 (CGMS) & $\mathrm{C}$ & 1 \\
\hline H. fagifolia (DC.) A. Juss. ${ }^{1}$ & Barbosa 1963 (ALCB) & $\mathrm{C}$ & 29 \\
\hline H. hatschabchii C.E. Anderson & Hatschbach 73989 (HUEFS) & $\mathrm{F}$ & 4 \\
\hline Janusia guaranitica (A. St.-Hil.) A. Juss. ${ }^{1,2}$ & Sebastiani 326 (SP) & $\mathrm{C}, \mathrm{F}, \mathrm{P}$ & 26 \\
\hline J. janusioides W.R. Anderson ${ }^{2}$ & Pott 6237 (SP) & $\mathrm{C}$ & 2 \\
\hline J. linearifolia A. St.-Hil.* & Hatschbach 74186 (MBM) & $\mathrm{C}$ & 1 \\
\hline J. mediterranea W.R. Anderson ${ }^{1,2}$ & Garcia 13896 (UEC) & $\mathrm{C}, \mathrm{F}$ & 1 \\
\hline J. occhionii W.R. Anderson ${ }^{1,2}$ & Pott 9733 (SP) & $\mathrm{C}, \mathrm{F}$ & 3 \\
\hline Mascagnia australis C.E. Anderson & Francener 1177 (SP) & $\mathrm{F}$ & 1 \\
\hline M. cordifolia (A. Juss.) Griseb. ${ }^{1,2}$ & Francener 875 (CGMS) & $\mathrm{C}, \mathrm{P}$ & 53 \\
\hline M. divaricata (Kunth) Nied. ${ }^{1,2}$ & Hatschbach 77349 (MBM) & $\mathrm{C}, \mathrm{P}$ & 14 \\
\hline M. sepium (A. Juss.) Griseb. ${ }^{1}$ & Araújo 1250 (CGMS) & $\mathrm{C}$ & 3 \\
\hline Niedenzuella multiglandulosa (A. Juss.) W.R. Anderson ${ }^{1}$ & Marques 1 (CGMS) & $\mathrm{F}$ & 1 \\
\hline N. sericea (A. Juss.) W.R. Anderson* & Tsuji 1320 (HPL) & $\mathrm{C}$ & 1 \\
\hline N. stannea (Griseb.) W.R. Anderson ${ }^{1,2}$ & Francener 806 (CGMS) & $\mathrm{C}, \mathrm{P}$ & 17 \\
\hline Peixotoa cordistipula A. Juss. ${ }^{2}$ & Pott 11295 (SP) & $\mathrm{C}, \mathrm{P}$ & 30 \\
\hline P. magnifica C. Anderson ${ }^{1,2}$ & Cecantini 2125 (SP) & $\mathrm{C}$ & 26 \\
\hline P. parviflora A.Juss. & Henschen $1652(\mathrm{~F})$ & $\mathrm{F}$ & 1 \\
\hline P. psilophylla C. Anderson ${ }^{2}$ & Jacques 773 (SP) & $\mathrm{C}$ & 1 \\
\hline P. reticulata Griseb. ${ }^{1,2}$ & Pott 8616 (SP) & $\mathrm{C}, \mathrm{P}$ & 43 \\
\hline P. tomentosa A. Juss.* & Boabaide 7 (SP) & $\mathrm{C}$ & 6 \\
\hline Ptilochaeta bahiensis Turcz. ${ }^{2}$ & Resende 545 (SP) & $\mathrm{C}$ & 1 \\
\hline P. densiflora Nied. ${ }^{1,2}$ & Damasceno Jr. 2642 (CGMS) & $\mathrm{C}, \mathrm{P}$ & 6 \\
\hline P. glabra Nied. $^{2}$ & Almeida Rego 5385 (SP) & $\mathrm{C}$ & 2 \\
\hline P. nudipes Griseb. * & Cláudio s.n. (RB) & $\mathrm{P}$ & 1 \\
\hline Stigmaphyllon calcaratum N. E. Br. ${ }^{1,2}$ & Francener 916 (CGMS) & $\mathrm{P}$ & 4 \\
\hline S. macedoanum C. Anderson* & Sebastiani 323(SP) & $\mathrm{C}, \mathrm{P}$ & 2 \\
\hline S. matogrossense C. Anderson * & Souza 1585 (HUEM) & $\mathrm{P}$ & 1 \\
\hline S. urenifolium A.Juss. & Anderson $9208(\mathrm{~F})$ & $\mathrm{C}$ & 1 \\
\hline Tetrapterys ambigua (A. Juss.) Nied. ${ }^{1,2}$ & Damasceno Jr. 924 (SP) & $\mathrm{C}$ & 10 \\
\hline T. crispa A. Juss. * & Boabaide2 (SP) & $\mathrm{F}$ & 1 \\
\hline T. hassleriana Nied. ${ }^{1}$ & Pott 7654 (SP) & $\mathrm{P}$ & 2 \\
\hline T. jussieuana Nied. $^{1,2}$ & Archer s.n. (SP) & $\mathrm{C}, \mathrm{P}$ & 5 \\
\hline T. phlomoides (Spreng) Nied.* & Pott 5494 (CPAP) & $\mathrm{F}$ & 1 \\
\hline T. racemulosa A. Juss. * & Caporal 1007 (CGMS) & $\mathrm{C}$ & 1 \\
\hline T. ramiflora A. Juss. ${ }^{1}$ & Pott 14586 (CGMS) & $\mathrm{C}$ & 8 \\
\hline T. turnerae A.Juss. & Pott 1512 (SP) & $\mathrm{P}$ & 2 \\
\hline Thryallis brachystachys Lindley * & Hatschbach 72948 (MBM) & $\mathrm{F}, \mathrm{P}$ & 2 \\
\hline T. laburnum S. Moore ${ }^{2}$ & Francener 941 (CGMS) & $\mathrm{P}$ & 13 \\
\hline T. latifolia Mart. $^{2}$ & Conceição 1232 (SP) & $\mathrm{C}$ & 5 \\
\hline
\end{tabular}




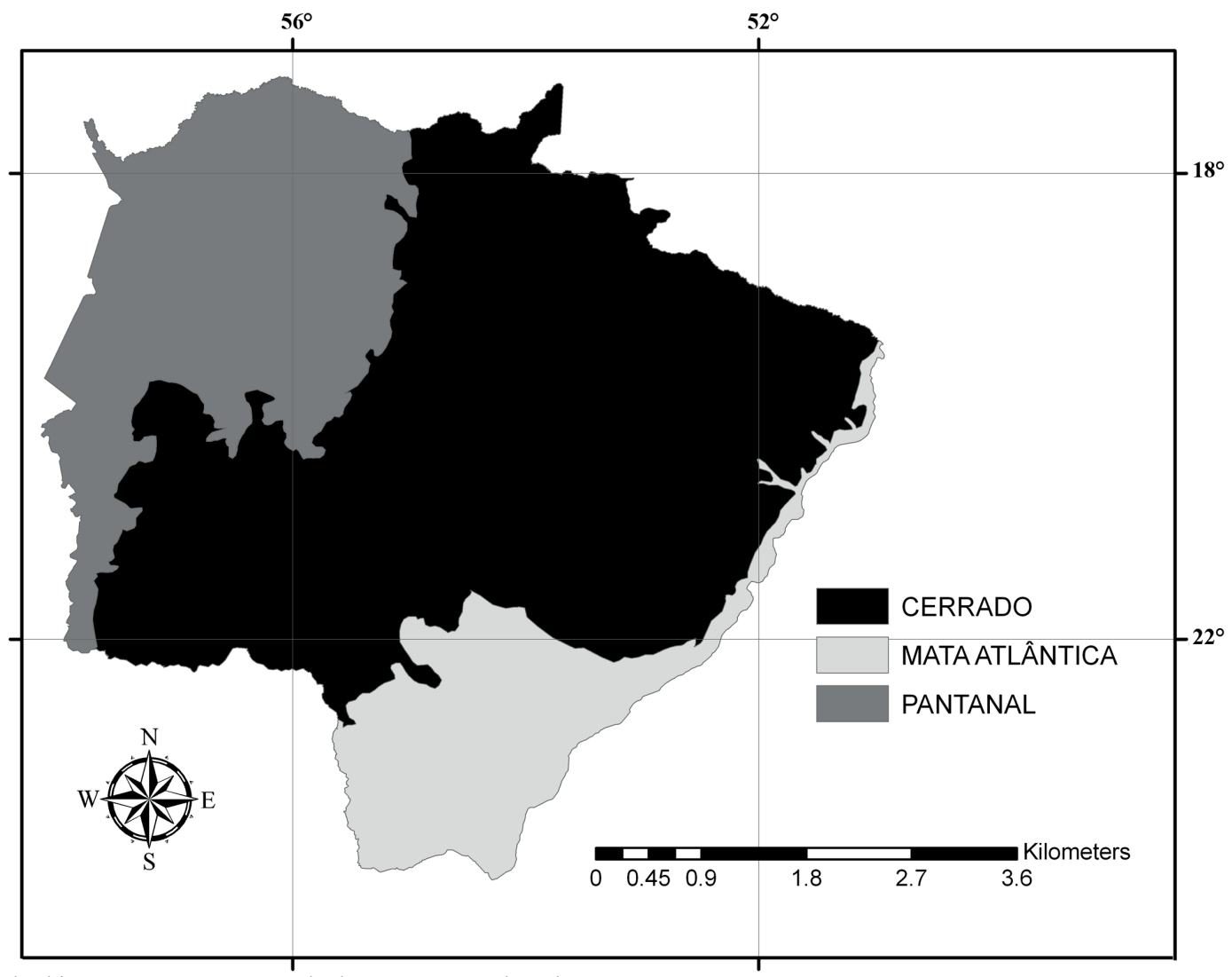

Fig.1. Mapa dos biomas ocorrentes no estado do Mato Grosso do Sul.

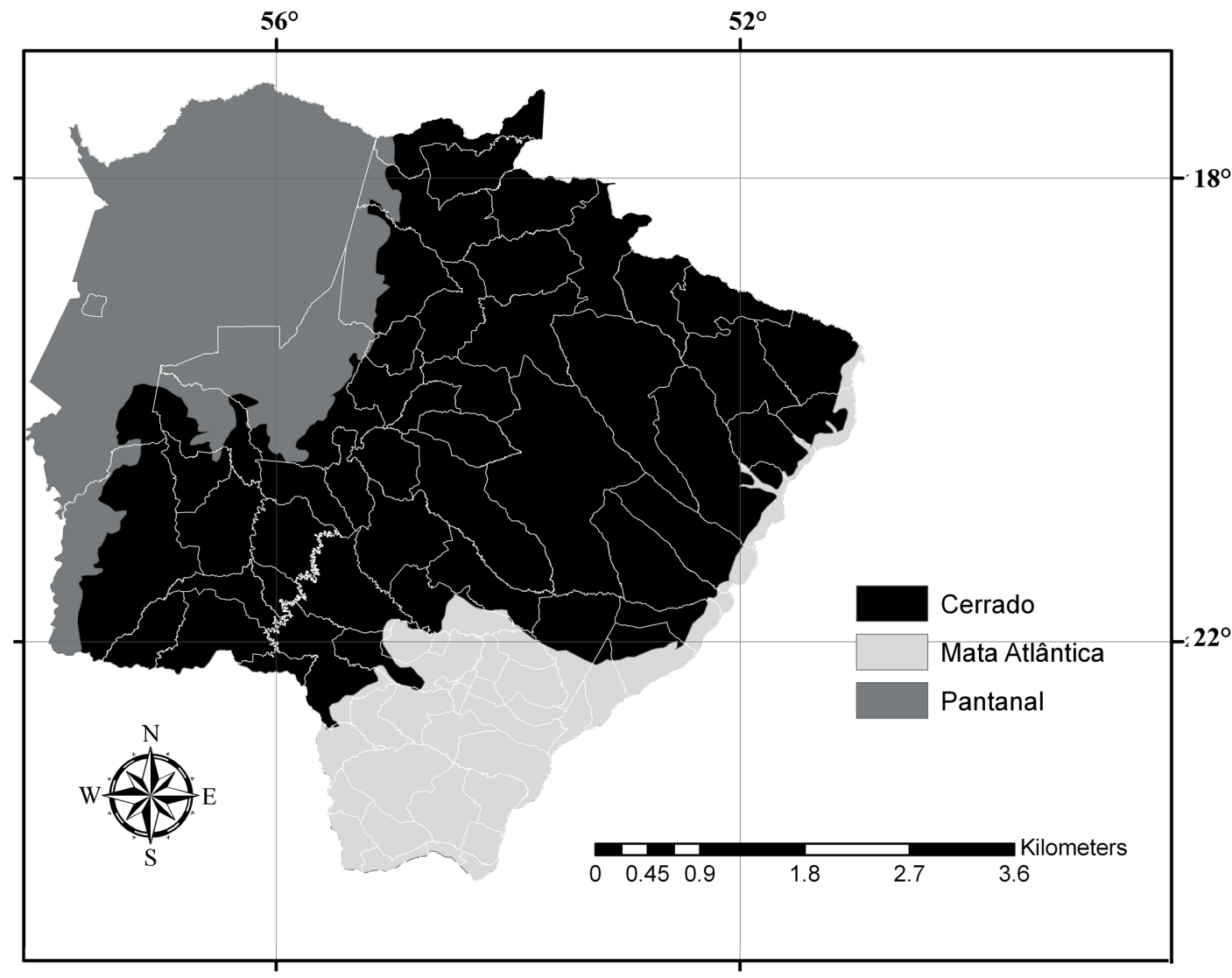

Fig. 2. Municípios do Mato Grosso do Sul classificados quanto aos biomas ocorrentes em seu território. 


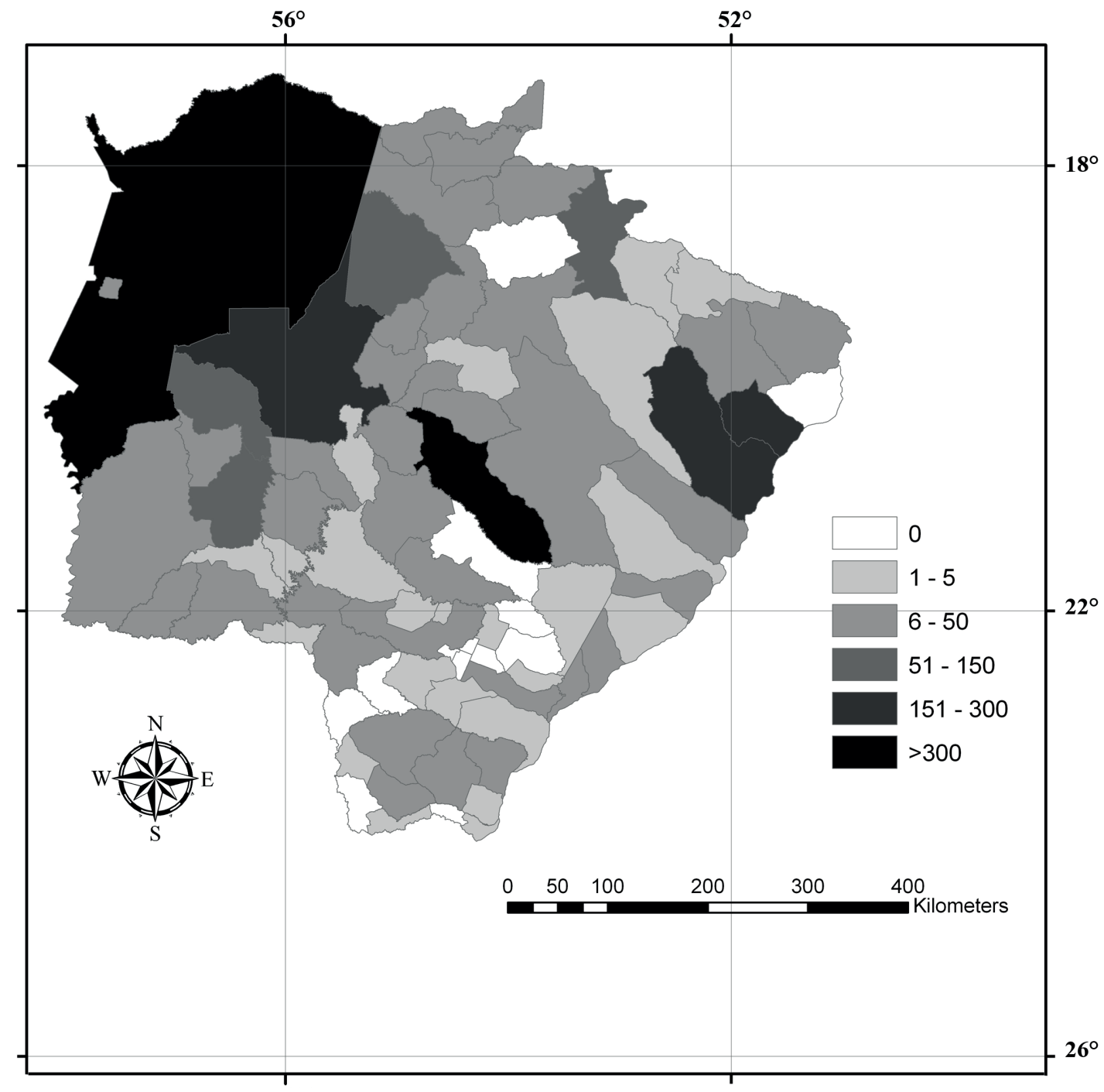

Fig. 3. Municípios do Mato Grosso do Sul classificados quanto ao número de coletas de espécimes de Malpighiaceae em seu território.

estado de São Paulo e ocorrência exclusiva em unidades de conservação e em um único tipo de formação vegetal ou ocorrência desconhecida em unidades de conservação, B. rigida, C. hirsuta H. cuiabensis. Por fim, B. pallescens e $T$. brachystachys são consideradas presumivelmente extintas no estado de São Paulo, devido à ausência de novos registros nos últimos 50 anos, inclusive em condições ex-situ. Apesar da lista de espécies ameaçadas usada para comparação pertencer ao estado de São Paulo, os dados aqui expostos refletem a importância do estudo da família Malpighiaceae para o Mato Grosso do Sul.

De acordo com Wanderley et al. (2012), o estado de São Paulo apresenta 108 espécies de Malpighiaceae, das quais 56 ocorrem também no estado do Mato Grosso do Sul. Anderson (1995) relatou a ocorrência de 18 espécies de Malpighiaceae no Pico das Almas, estado da Bahia, das quais metade é relatada para o estado do Mato Grosso do Sul. O check-list para as Malpighiaceae da Chapada dos Veadeiros, Sebastiani et al. (2015) identificaram cerca de
80 espécies, a maior parte delas em comum para as áreas de cerrado em Mato Grosso do Sul aqui apresentadas. Mamede (1987) relatou 43 espécies para a Flora da Serra do Cipó em Minas Gerais, sendo destas 23 em comuns com a flora do MS. Já para a Flora de Grão Mogol, em Minas Gerais, 26 espécies foram registradas, sendo 16 também ocorrentes para a área de estudo (Mamede 2004). Assim, é notável a diversidade de Malpighiaceae para o estado do Mato Grosso do Sul, revelada no presente estudo.

\section{AGRADECIMENTOS}

Os autores agradecem aos curadores dos herbários visitados e a Alice Francener da Universidade Federal de Goiás pelo auxílio na elaboração dos mapas. Agradecemos também a bolsa de doutorado concedida ao segundo autor pela Fundação de Amparo à Pesquisa do estado da Bahia (584/2013), ao financiamento dos estudos de campo e visitas à herbários através dos projetos Reflora 
Malpighiales (Coordenação de Aperfeiçoamento de Pessoa de Nível Superior, Processo 563548/2010-0) e Universal Malpighiales (Conselho Nacional de Desenvolvimento Científico e Tecnológico, Processo 486079/2013-9).

\section{REFERÊNCIAS}

Alexandrino, V.H.D., Sousa, J.S. \& Bastos, M.N.C. 2011. Estudo taxonômico da família Malpighiaceae Juss. das restingas de Algodoal/ Maiandeua, Maracanã, Pará, Brasil. Boletim do Museu Paraense Emílio Goeldi. Ciências Naturais 6(3):335-347.

Almeida, R.F. 2015. New records of Stigmaphyllon puberulum Griseb. (Malpighiaceae) from the Atlantic Forest, northeastern Brazil. Check List 11:1510-1512.

Almeida, R.F. \& Amorim, A.M. 2014a. Stigmaphyllon caatingicola (Malpighiaceae), a new species from Seasonally Dry Tropical Forests in Brazil. Phytotaxa 174:82-88.

2014b. Malpighiaceae. In Plantas Vasculares do Paraná. (M. Kaehler, R. Goldenberg, P.H. Labiak, O.S. Ribas, A.O.S. Vieira, G.G. Hatschbach, eds.). Editora Universidade Federal do Paraná. p. 131-132.

2015. Stigmaphyllon mikanifolium (Malpighiaceae), a new species for Espírito Santo State, Brazil. Kew Bulletin 70(47):1-7.

Almeida, R.F. \& Mamede, M.C.H. 2014. Checklist, conservation status, and sampling effort analysis of Malpighiaceae in Espírito Santo state, Brazil. Brazilian Journal of Botany 37:329-337.

Sinopse de Malpighiaceae no estado do Espírito Santo: Stigmaphyllon A. Juss. (Hoehnea, submetido).

Almeida, R.F. \& Pellegrini, M.O.O. 2016. Synopsis of Bunchosia Kunth (Malpighiaceae) from the Atlantic Forest. Phytotaxa 257(2): 158-166.

Almeida, R.F., Dal Col, A.C. \& Amorim, A.M.A. 2015. Notes on Stigmaphyllon (Malpighiaceae) from Southeastern Brazil. Boletim do Museu de Biologia Mello Leitão (Nova Série) 37(4):427-436.

Almeida, R.F., Francener, A. \& Amorim, A.M.A. 2016. A generic synopsis of Malpighiaceae Juss. in the Atlantic Forest. Nordic Journal of Botany, early view.

Almeida, R.F., Francener, A. \& Sebastiani, R. 2013. New records on endangered and endemic species of Stigmaphyllon A. Juss (Malpighiaceae) in Brazil. Check List. 9(5):1084-1086.

Almeida, R.F., Francener, A., Pessoa, C.S. \& Amorim, A.M.A. 2015. Malpighiaceae I: chave para gêneros. In Prata, A.P.N. et al. (eds) Flora do Sergipe, volume II. Aracaju: Editora Criação. p.191-193.

Amorim, A.M. 1994. Malpighiaceae. In Reserva Ecológica de Macaé de Cima. (R. Guedes-Bruni, M.P.M. Lima, eds.), Jardim Botânico do Rio de Janeiro, p. 229-250.

Amorim, A.M. 2002. Five new species of Heteropterys (Malpighiaceae) from Central and South America. Brittonia 54(4):217-232.

2003. Estudos taxonômicos em Heteropterys (Malpighiaceae). Tese 286 f., Universidade de São Paulo, São Paulo.

Amorim, A.M.A. 2004. A new species of Heteropterys (Malpighiaceae) from the semideciduous forest of Bahia, Brazil. Brittonia 56(2):143146.

Amorim, A.M.A. 2005. Heteropterys jardimii (Malpighiaceae), uma nova espécie para a Bahia, Brasil. Rodriguésia 56(87):175-178.

Amorim, A.M.A. \& Almeida, R.F. 2015. An unexpected Mcvaughia (Malpighiaceae) species from Sandy Coastal Plains in Northeastern Brazil. Systematic Botany 40(2):1-5.

Amorim, A.M.A. \& São-Mateus, W.M.B. 2009. Redescrição de Heteropterys bahiensis (Malpighiaceae). Rodriguésia 60 (2):367-370.

Anderson, C. 1982. A monograph of the genus Peixotoa (Malpighiaceae). Contributions of the University of Michigan Herbarium 15:1-92.

1995. Revision of Thryallis (Malpighiaceae). Contributions of the University of Michigan Herbarium 20: 3-14.

1997a. Monograph of Stigmaphyllon (Malpighiaceae). Systematic Botany Monographs 51: 1-313.

1997b. Revision of Pterandra (Malpighiaceae). Contributions from the University of Michigan Herbarium 21:1-27.

2001. Novelties in Mascagnia (Malpighiaceae). Brittonia $53: 405-415$.
2005. Pterandra viridiflora (Malpighiaceae), a new species from Brazil. Contributions of the University of Michigan Herbarium 24:21-23.

2007. Revision of Galphimia (Malpighiaceae). Contributions of the University of Michigan Herbarium 25:1-82.

2009. Stigmaphyllon lanceolatum (Malpighiaceae), a new species from Espírito Santo, Brazil. Harvard Papers on Botany 14:193-196.

2011. Revision of Ryssopterys and transfer to Stigmaphyllon (Malpighiaceae). Blumea 56:73-104.

2013a. Six new species of Hiraea (Malpighiaceae) from South America: $H$. andersonii, $H$. brevistipulata, $H$. holmgreniorum, $H$. kariniana, $H$. singularis, and $H$. woytkowskii. Memoirs of the New York Botanical Garden 108:205-221.

2013b. Resolution of the Hiraea cephalotes complex (Malpighiaceae). Edinburgh Journal of Botany 70:413-432.

2014. Hiraea cuneata, $H$. macrophylla, and four new species confused with them: $H$. hatschbachii, $H$. occhionii, H. reitzii, and $H$. restingae (Malpighiaceae). Edinburgh Journal of Botany 73:361-378.

Anderson, W.R. 1979. Floral conservatism in neotropical Malpighiaceae. Biotropica 11(3):219-223.

1981. Malpighiaceae. The botany of the Guayana HighlandPart XI. (B. Maguire, ed.) Memoirs of New York Botanical Garden 32:21-305.

1982. Notes on Neotropical Malpighiaceae I. Contributions of the University of Michigan Herbarium 15:93-136. 1993. Notes on Neotropical Malpighiaceae IV. Contributions of the University of Michigan Herbarium 19:355-392. 1995. Malpighiaceae In Flora of the Pico das Almas, Chapada

Diamantina (B.L. Stannard, ed.). Bahia, Brazil. pp. 416-430. 1997. Notes on Neotropical Malpighiaceae VI. Contributions of the University of Michigan Herbarium 21:37-84. 1998. Malpighiaceae In Prodromus Florae Matogrossensis. (B.

Dubs ed.) Part 1. Checklist of Angiosperms, p. 176-185. 1999. Notes on neotropical Mapighiaceae VII. Contributions of the University of Michigan Herbarium 22:1-19. 2006. Eight segregates from the neotropical genus Mascagnia (Malpighiaceae). Novon 16:168-204.

2014. Seven new species of neotropical Malpighiaceae. Acta Botanica Mexicana 109:23-43.

\& Anderson. C.E. 2015. Three new species of Bunchosia (Malpighiaceae): B. cuscana, B. hedraiophylla, and B. neillii. Brittonia 67:243-249.

\& Davis, C.C. 2005. The Mascagnia cordifolia group (Malpighiaceae). Contributions of the University of Michigan Herbarium 24:33-44.

2006. Expansion of Diplopterys at the expense of Banisteriopsis

(Malpighiaceae) Harvard Papers in Botany. 11(1):1-16. 2007. Generic adjustments in neotropical Malpighiaceae.

Contributions of the University of Michigan Herbarium 25:137-166. 2012. Proposal to conserve the name Mascagnia against

Triopterys (Malpighiaceae). Taxon 61:1124-1125.

2013. Combination of Mascagnia and Triopterys. Memoirs of the New York Botanical Garden 108:191-203.

\& Gates, B. 1981. Barnebya, a new genus of Malpighiaceae from Brazil. Brittonia 33(3):275-284.

BFG - The Brazilian Flora Group. 2015. Growing knowledge: an overview of Seed Plant diversity in Brazil. Rodriguésia 66(4): 1085-1113.

Carvalho, P.D., Rapini, A. \& Conceição, A.A. 2010. Flora da Bahia: Malpighiaceae I - Banisteriopsis, Bronwenia e Diplopterys. Sitientibus 10:159-190.

Conceição, G.M., Ruggieri, A.C. \& Rodrigues, M.S. 2011. Malpighiaceae do cerrado do Parque Estadual do Mirador, Maranhão, Brasil. Scientia Plena 7(2):1-6.

Davis, C.C., Bell, C.D., Matheus, S. \& Donoghue, M.J. 2002. Laurasian migration explains gondwanan disjunctions: evidence from Malpighiaceae. Proceedings of the National Academy of Sciences 99(10):6833-6837.

Davis, C.C. \& Anderson, W.R. 2010. A complete generic phylogeny of Malpighiaceae inferred from nucleotide sequence data and morphology. American Journal of Botany 97(12):2031-2048. 
Elias, S.I. 2005. Revisão e redefinição de Byrsonima Rich. ex Kunth subg. Macrozeugma Nied. (Malpighiaceae). Tese 371 f., Universidade de São Paulo, São Paulo.

Francener, A., Damasceno-Júnior, G.A., Gomes-Klein, V.L. 2015. Tetrapterys Cav. (Malpighiaceae) from Brazilian Midwest. Acta Botanica Brasílica 29(1):143-156.

Francener, A. 2016. Estudos taxonômicos em Byrsonima sect. Eriolepsis Nied. (Malpighiaceae). Tese 184f. Instituto de Botânica, São Paulo.

Gates, B. 1982. Banisteriopsis, Diplopterys (Malpighiaceae). Flora Neotropica Monograph 30:1-237.

Gomes-Klein, V.L. \& Rizzo, J.A. 2006. Estudos sobre a flora do CentroOeste do Brasil: situação atual e perspectivas. In Os avanços da botânica no início do século XXI. (J.E.A. Mariath, \& R.P. Santos), Porto Alegre, Anais do $57^{\circ}$ Congresso Nacional de Botânica, Sociedade Botânica do Brasil. 752p.

Lima, B.C.S., Almeida, R.F. \& Prata, A.P.N. 2015. Malpighiaceae IStigmaphyllon. In Flora do Sergipe (A.P.N. Prata, M.C. Vieira, M.F. Landin, eds.). Criação Editora, p. 193-202.

Mamede, M.C.H. 1981. O gênero Byrsonima Rich. ex A. Juss. (Malpighiaceae) na Serra do Cipó, Minas Gerais, Brasil. Dissertação 245 f., Universidade de São Paulo, São Paulo.

. 1984. Flora fanerogâmica da Reserva do Parque Estadual das Fontes do Ipiranga (São Paulo, Brasil). 125. Malpighiaceae. Hoehnea 11:108-113.

1987. Flora da Serra do Cipó, Minas Gerais: Malpighiaceae. Boletim de Botânica da Universidade de São Paulo 9:157-198. 1990. Revisão do gênero Camarea Saint-Hilaire (Malpighiaceae). Hoehnea 17(1):1-34.

1992. Malpighiaceae In Flora fanerogâmica da Ilha do Cardoso (M.M.R.F. Melo, F. Barros, S.A.C. Chiea, M.G.L. Wanderley, S.L. Jung-Mendaçolli, M. Kirizawa org.) Instituto de Botânica, São Paulo, v. 3, p. 73-87.

2004. Flora de Grão-Mogol, Minas Gerais: Malpighiaceae.

Boletim de Botânica da Universidade de São Paulo 22(2):291-302. . Souza, V.C., Prado, J., Barros, F., Wanderley, M.G.L. \& Rando, J.G. 2007. Livro vermelho das espécies vegetais ameaçadas do estado de São Paulo. Instituto de Botânica, São Paulo. 165 p. . Amorim, A.M.A. \& Sebastiani, R. 2010. Malpighiaceae In Catálogo de plantas e fungos do Brasil (R.C. Forzza, C.E.M. Bicudo, J.R. Carvalho JR., A.A. Costa, D.P. Costa, M. Hopkins, P.M. Leitman, L.G. Lohmann, L.C. Mais, G. Martinelli, M. Menezes, M.P. Morim, M.A. Nadruz Coelho, A.L. Peixoto, J.R. Pirani, J. Prado, L.P. Queiroz, V.C. Souza, J.R. Stehmann, L.S. Sylvestre, B.M.T. Walter, \& D. Zappi, D., org.). v. 2. Andrea Jakobsson Estúdio. Instituto de Pesquisas Jardim Botânico do Rio de Janeiro. Rio de Janeiro, p. 1183-1201.

Ministério do Meio Ambiente - MMA. 2007. Mapa de cobertura vegetal dos biomas brasileiros. $18 \mathrm{p}$
Ministério do Meio Ambiente - MMA. 2012. Mapas de cobertura vegetal dos biomas brasileiros. Disponível em: http://mapas.mma.gov.br/ mapas/aplic/probio/datadownload.htm. Acessado em 30.08.2012.

Mittermeier, R.A., Gil, P.R., Hoffman, M., Pilgrim, J., Brooks, T., Mittermeier, C.G., Lamoureux, J. \& Fonseca, G.A.B. 2005. Hotspots revisited: earth's biologically richest and most endangered terrestrial ecoregions. Conservation International, Washington. 392 p.

Niedenzu, F. 1928. Malpighiaceae. In Engler A. Das Pflanzenreich. Wilhelm Engelmann. Leipzig v. 141, 870 p.

Pessoa, C., Costa, J.A.S. \& Amorim, A.M.A. 2014. Flora da Bahia: Malpighiaceae 2 - Heteropterys. Sitientibus 14:1-41.

Pessoa, C.S. \& Amorim, A.M.A. 2016. Heteropterys arcuata (Malpighiaceae): a new species from the dry forests of northeastern Brazil. Phytotaxa 260(1): 83-88.

Sebastiani, R. 2010. Estudos taxonômicos em Janusia A. Juss. (Malpighiaceae). Tese 177 f., Instituto de Botânica da Secretaria do Meio Ambiente, São Paulo.

Sebastiani, R. \& Mamede, M.C.H. 2010. Estudos taxonômicos em Heteropterys Kunth subsect. Stenophyllarion (Malpighiaceae) no Brasil. Hoehnea. 37(2):337-366.

2014. Two new species of Janusia (Malpighiaceae) from Brazil. Hoehnea 41(1):121-127.

Sebastiani, R., Francener, A., Silva, C.V. \& Almeida, R.F. 2015. An Illustrated Checklist of Malpighiaceae from Chapada dos Veadeiros region, Goiás, Brazil. Check List. 11:1801-1809.

Silva, D.S.P. \& Almeida, R.F. 2015. Malpighiaceae. Catálogo das espécies de plantas vasculares e briófitas do Estado do Rio de Janeiro. Rio de Janeiro: Instituto de Pesquisas Jardim Botânico do Rio de Janeiro. Disponível em: http://florariojaneiro.jbrj.gov.br. Acessado em 26.09.2015.

SpeciesLink. 2016. Disponível em: http://splink.cria.org.br. Acessado em 24.05.2016.

Thiers, B. 2016. Index Herbariorum: A global directory of public herbaria and associated staff. New York Botanical Garden's Virtual Herbarium. Disponível em: http://sciweb.nybg.org/science2/IndexHerbariorum. asp. Acessado em 24.05.2016.

Vicentini, A. 1999. Malpighiaceae. In Flora da Reserva Ducke (J.E.L.R Ribeiro, M.J.G. Hopkins, A. Vicentini, C.A. Sothers, M.A.S. Costa, J.M. Brito, M.A.D. Souza, L.H.P. Martins, L.G. Lohmann, P.A.C.L. Assunção, E.C. Pereira, C.R. Silva, M.R. Mesquita \& L. Procópio eds.), Editora Instituto Nacional de Pesquisas da Amazônia, Manaus, p. 505-511.

Wanderley, M.G.L., Shepherd, G.J., Martins, S.E., Estrada, T.EM.D., Romanini, R.P., Koch, I., Pirani, J.R., Melhem T.S., Harley, A.M.G., Kinoshita, L.S., Magenta, M.A.G., Wagner, H.M.L., Barros, F., Lohmann, L.G., Amaral, M.C.E., Cordeiro, I., Aragaki, S., Bianchini, R.S. \& Esteves, G.L. 2012. Checklist das Spermatophyta do Estado de São Paulo, Brasil. Biota Neotropica 11(1a):193-390. 